\title{
Partner notification services of the Sexually Transmitted Disease clinics in Sri Lanka
}

\author{
Wijayawickrama D. $\mathrm{N}^{1}$, Ariyaratne K.A. Manathunge ${ }^{2}$
}

\begin{abstract}
Introduction: Partner notification is a strategy used to control sexually transmitted infections (STI). It includes identifying a look-back interval and offering testing and treatment to the relevant sexual partners of an index patient.
\end{abstract}

Objective: Study was carried out to assess partner notification (PN) services at selected Sexually Transmitted Disease (STD) clinics.

Method: Study consisted of four components. Component 1 was a cross sectional descriptive study among the staff of five selected STD clinics who were directly involved in PN, to assess knowledge, attitudes and practices on PN. Component 2 was a retrospective descriptive study in the same setting to describe the burden of partner notifiable STIs during 2012. Component 3 was a casecontrol study among patients with partner notifiable STIs in the STD clinic, Colombo during 2012 to describe factors associated with patient compliance in PN. Component 4 was a cross sectional study at STD clinic, Colombo to evaluate PN indices.

Results: All interviewed staff knew that gonorrhoea, early syphilis and chlamydia infection are partner notifiable but some misidentified bacterial vaginosis (20\%) and vaginal candidiasis (14\%) as partner notifiable. Knowledge on contact actions and look back period were unsatisfactory and $17 \%$ and $10 \%$ respectively were unaware that look back period is irrelevant to genital herpes and genital warts. A total of 1,157 partner notifiable STIs have been reported from all five clinics in 2012. Patient compliance in PN was significantly associated with regular partners, NGI and coitarche $\geq 19$ years. Percentages of index cases interviewed for PN and index cases who had documented outcomes for all contacts were only $30.7 \%$ and $8.1 \%$ respectively.

Conclusions: Knowledge, attitude and practices on PN among the clinic staff was unsatisfactory. Percentage of index cases interviewed was low (30\%) probably leaving a considerable number of contacts unattended. Patient compliance on PN was significantly associated with regular partners, coitarche $\geq 19$ years and NGI. Documentation relevant to PN was unsatisfactory.

Key words: Partner notification, Sri Lanka, Sexually Transmitted infections

\footnotetext{
Authors: corresponding author; ${ }^{1}$ Dr D. N. Wijayawickrama, MBBS, PgDVen, MD, Consultant Venereologist, Teaching Hospital, Galle.Email: drashani.wijewickrama@gmail.com, iD https://orcid.org/0000-0002-5920-4919

${ }^{2} \mathrm{Dr}$ Ariyaratne $\mathrm{K}$ A Manathunge, MBBS, MSC, MD, Consultant Venereologist, National STD/AIDS Control Programme, Colombo. Email: ariyaratne1@gmail.com, iD https://orcid.org/0000-0001-9514-9227

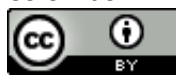

Acknowledgement: All the participants in the study, my colleagues who helped in this study Conflict of interest: Authors claim no conflicts of interest, Funding: not funded, Originality: This is an original work which has been not published anywhere else, Submitted: 25.11.2017, Accepted:11.12.2017
} 


\section{Full article}

\section{Introduction}

Partner notification (contact tracing) is a strategy used to control STIs. It involves identifying a look-back interval in which infection of contacts may have occurred, informing the relevant contacts of their exposure, offering testing and treatment and recording contact actions and outcomes. (1) Following contact actions are used in partner notification.

Patient referral: Patients are encouraged to notify partners at risk.

Provider referral: Health care workers assist in notifying partners.

Contract referral: A time frame is negotiated by the provider, for the patient to notify partners. (2)

No action: This is appropriate when a contact is non-traceable or has been verified as already visited a health-care facility. (1)

Gonorrhoea, syphilis, NGI and trichomoniasis were considered as partner notifiable infections (3) in this study.

\section{Partner notification process in Sri Lanka:}

The medical officer (MO) refers the relevant patient to the public health inspector (PHI) or the public health nursing sister (PHNS) for partner notification interview to identify primary contacts of the index patient.
All possible details of the contacts are recorded in

\section{- Patient's record}

- Interview and contact tracing register

- Counselling and partner notification form (H-1205)

Contact action is determined with the informed consent of the index patient. Contact slips are issued (3) if patient referral is the agreed action.

This study was performed to understand partner notification among patients with STIs in Sri Lanka since no such study has been done so far STD clinics at Colombo, Ragama, Gampaha, Kalutara and Kandy were selected as study setting since it was assumed that Patient Information Management System (PIMS) implemented in those, will enable gathering data more systematically.

The objective of the study was to assess the level of partner notification in selected STD clinics and to understand the knowledge, attitudes and practices of health care workers relevant to partner notification and factors associated with compliance with partner notification.

\section{Methods}

The study had four components with different study designs as outlined below. (Table 1)

Table 1: Study components

\begin{tabular}{|c|c|c|c|}
\hline \multicolumn{2}{|c|}{ Description of the component } & Study setting & Population/Sampling technique \\
\hline 1 & $\begin{array}{l}\text { Cross sectional descriptive study among MOs, } \\
\text { PHIs, PHNSs and nursing officers directly } \\
\text { involved in partner notification from } 25^{\text {th }} \\
\text { October- } 25^{\text {th }} \text { November, } 2013\end{array}$ & $\begin{array}{l}\text { STD clinics at } \\
\text { Colombo, Ragama, } \\
\text { Kandy, Kalutara and } \\
\text { Gampaha }\end{array}$ & All staff \\
\hline 2 & $\begin{array}{l}\text { Cross sectional study to describe the burden of } \\
\text { partner notifiable STIs reported during the } \\
\text { year } 2012\left(1^{\text {st }} \text { January to } 31^{\text {st }} \text { December }\right)\end{array}$ & $\begin{array}{l}\text { Five selected STD } \\
\text { clinics }\end{array}$ & $\begin{array}{l}\text { All consecutive newly diagnosed patients } \\
\text { with partner notifiable STIs }\end{array}$ \\
\hline 3 & $\begin{array}{l}\text { Case control study to determine the factors } \\
\text { associated with compliance with partner } \\
\text { notification among index patients during the } \\
\text { year } 2012\left(1^{\text {st }} \text { January to } 31^{\text {st }} \text { December }\right) \text {. }\end{array}$ & STD clinic, Colombo & $\begin{array}{l}\text { All new patients with partner notifiable } \\
\text { STIs, Study sample: All above patients } \\
\text { who were interviewed at least once for } \\
\text { partner notification }\end{array}$ \\
\hline 4 & $\begin{array}{l}\text { Secondary data analysis to evaluate partner } \\
\text { notification services in year } 2012 \text { (from } \\
1^{\text {st }} \text { January to } 31^{\text {st }} \text { December). }\end{array}$ & STD clinic, Colombo & $\begin{array}{l}\text { All consecutive newly diagnosed patients } \\
\text { with partner notifiable STIs }\end{array}$ \\
\hline
\end{tabular}


Data collection: Data collection for the four components was done using three different methods.

Data collection from the clinic staff

(Component 1) was done using a structured self-administered questionnaire which included questions on their sociodemographic information, basic and essential knowledge, attitudes and practices on partner notification.

Data on partner notifiable STIs (Component 2) were extracted from the PIMS.

Data for the case control part (Component 3) was obtained by a data extraction sheet structured to extract data from PIMS, patients' records, $\mathrm{H}-1205$ and interview and contact tracing register.

Registration numbers of new patients with partner notifiable STIs were obtained from main register and the data were collected using the data extraction sheet.

Data collection on partner notification of the index patients attended during 2012 was continued for further six months (till the $31^{\text {st }}$ of June 2013). It was assumed that all reasonable steps to notify partners have been taken by that time.

Evaluation of partner notification services at STD clinic, Colombo (component 3) was done using the same data extraction sheet developed for the component 2 . In addition, data on treatment status of the contact was gathered from the clinic record of the contact. Date of data entry in to $\mathrm{H}-1205$ was considered as the date of interview of the index patient as there was no separate place to enter the date of interview. This was important in calculating the time taken for the contact to come to the relevant STD clinic following partner notification interview.

Patients were considered as interviewed, when the particular patient's clinic record contained filled (H-1205). There were 229 interviewed patients. Cases and controls were selected out of those as below.

Cases: Interviewed index patients of whom at least one partner attended the clinic

Controls: Interviewed index patients of whom at least one partner did not attend the clinic Following formula was used to calculate the sample size for the case control analysis. (4)

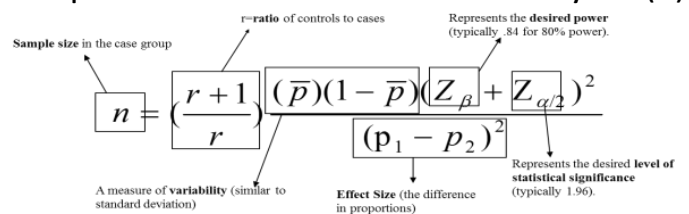

$\mathrm{P}_{1}$ (proportion of exposure among cases) $=$ 26\%

$\mathrm{P}_{2}$ (proportion of exposure among controls $)=$ $9 \%$.

$r=2$ (ratio of cases: controls $=1: 2$ )

Level of significance $=0.05$.

The number of cases ( $n$ ) was calculated as 57 and $5 \%$ added for missing data. Final sample size was 60 . Since the ratio of cases to controls was taken as 1:2, number of controls needed was 120. However, there were 169 patients with partner notifiable STIs who met the eligibility criteria as controls and they all were included in the analysis to further increase the power of the statistical analysis. Data were entered in to the SPSS version 13 for descriptive statistical analysis.

\section{Results}

Participants (22 MOs and 10 public health staff officers) represented $70 \%$ of all staff attached to these five clinics.

Majority were males. Over $90 \%$ were in the age group of 30-53 years, and over half of them had 1-5years of service experience (Table 2)

The table 3 contains the questions and the proportions of staff that gave the correct answers. 
Table 2. Sample characteristics

\begin{tabular}{|c|c|c|c|}
\hline Variable & Levels & Freq & Percent (\%) \\
\hline \multirow[t]{3}{*}{ Sex } & Male & 12 & 37.5 \\
\hline & Female & 20 & 62.5 \\
\hline & Total & 32 & 100.00 \\
\hline \multirow[t]{7}{*}{ Age } & $24-29$ & 1 & 3.1 \\
\hline & $30-35$ & 7 & 21.9 \\
\hline & $36-41$ & 10 & 30.0 \\
\hline & $42-47$ & 6 & 18.8 \\
\hline & $48-53$ & 6 & 18.8 \\
\hline & $54-59$ & 2 & 6.3 \\
\hline & Total & 32 & 100.00 \\
\hline \multirow[t]{6}{*}{ Work experience } & $<3 m$ & 0 & 0.0 \\
\hline & $\geq 3 m<1 y$ & 4 & 12.5 \\
\hline & $\geq 1 y<5 y$ & 19 & 59.4 \\
\hline & $\geq 5 y<10 y$ & 5 & 15.6 \\
\hline & $\geq 10 y$ & 4 & 12.5 \\
\hline & Total & 32 & 100.00 \\
\hline
\end{tabular}

Table 3: knowledge on partner notificationby staff category

\begin{tabular}{|c|c|c|c|c|c|c|c|}
\hline \multirow[t]{2}{*}{ Variable/ Question } & \multirow[t]{2}{*}{ Variable level } & \multicolumn{2}{|r|}{ MOs } & \multicolumn{2}{|c|}{$\begin{array}{r}\text { Public health } \\
\text { staff }\end{array}$} & \multicolumn{2}{|c|}{ All staff } \\
\hline & & $\mathrm{n} / \mathrm{N}$ & $\%$ & $\mathrm{n} / \mathrm{N}$ & $\%$ & $\mathrm{n} / \mathrm{N}$ & $\%$ \\
\hline \multirow[t]{3}{*}{$\begin{array}{l}\text { Knowledge on basics by } \\
\text { the staff categories }\end{array}$} & $\begin{array}{l}\text { Every patient with a STD should be } \\
\text { interviewed for contact tracing }\end{array}$ & $11 / 22$ & 50.0 & $4 / 10$ & 40.0 & $15 / 32$ & 42.8 \\
\hline & $\begin{array}{l}\text { Public health staff should maintain an } \\
\text { interview and contact tracing register in } \\
\text { clinic }\end{array}$ & $22 / 22$ & 100.0 & $9 / 10$ & 90.0 & $31 / 32$ & 96.8 \\
\hline & $\begin{array}{l}\text { Person/persons responsible for contact } \\
\text { tracing }\end{array}$ & $8 / 22$ & 34.3 & $5 / 10$ & 50.0 & $13 / 32$ & 40.6 \\
\hline \multirow{7}{*}{$\begin{array}{l}\text { Which of the following } \\
\text { are methods of contact } \\
\text { tracing? }\end{array}$} & Sending a letter to the contact & $10 / 21$ & 47.6 & $4 / 9$ & 44.4 & $14 / 30$ & 46.7 \\
\hline & $\begin{array}{l}\text { Giving a confidential phone call to the } \\
\text { contact }\end{array}$ & $12 / 19$ & 63.2 & $5 / 9$ & 55.6 & $17 / 28$ & 60.7 \\
\hline & Visiting the contact privately & $10 / 20$ & 50.0 & $7 / 9$ & 77.8 & $17 / 29$ & 58.6 \\
\hline & Asking the patient to bring the contact & $21 / 21$ & 100.0 & $10 / 10$ & 100.0 & $31 / 31$ & 100.0 \\
\hline & $\begin{array}{l}\text { Contacting the partner directly if } \\
\text { provider referral failed }\end{array}$ & $8 / 21$ & 38.1 & $5 / 8$ & 62.5 & $13 / 29$ & 44.8 \\
\hline & $\begin{array}{l}\text { Treating the contact through the } \\
\text { patient }\end{array}$ & $15 / 20$ & 75.0 & $4 / 9$ & 4.4 & $19 / 29$ & 65.5 \\
\hline & $\begin{array}{l}\text { Asking a friend of the contact to bring } \\
\mathrm{him} / \text { her to the STD clinic }\end{array}$ & $15 / 20$ & 75.0 & $9 / 9$ & 100.0 & $26 / 29$ & 89.6 \\
\hline \multirow{12}{*}{$\begin{array}{l}\text { Which infection needs } \\
\text { contact tracing? }\end{array}$} & Gonococcal urethritis & $22 / 22$ & 100.0 & $10 / 10$ & 100.0 & $32 / 32$ & 100.0 \\
\hline & Gonococcal cervicitis & $22 / 22$ & 100.0 & $10 / 10$ & 100.0 & $32 / 32$ & 100.0 \\
\hline & Vaginal candidiasis & $20 / 22$ & 90.9 & $5 / 7$ & 71.4 & $29 / 25$ & 86.2 \\
\hline & Primary syphilis & $22 / 22$ & 100.0 & $10 / 10$ & 100.0 & $32 / 32$ & 100.0 \\
\hline & Secondary syphilis & $22 / 22$ & 100.0 & $10 / 10$ & 100.0 & $32 / 32$ & 100.0 \\
\hline & Early latent syphilis & $22 / 22$ & 100.0 & $7 / 9$ & 77.7 & $29 / 31$ & 93.5 \\
\hline & Late syphilis & $13 / 22$ & 59.1 & $6 / 9$ & 66.7 & $19 / 31$ & 61.3 \\
\hline & Genital herpes & $15 / 22$ & 68.2 & $4 / 9$ & 44.4 & $19 / 31$ & 61.3 \\
\hline & Genital warts & $17 / 22$ & 77.3 & $3 / 5$ & 37.5 & $20 / 30$ & 66.7 \\
\hline & Chlamydia infection & $22 / 22$ & 00.0 & $10 / 10$ & 100.0 & $30 / 30$ & 100.0 \\
\hline & Trichomoniasis & $22 / 22$ & 00.0 & $9 / 10$ & 90.0 & $31 / 32$ & 96.8 \\
\hline & Bacterial vaginosis & $20 / 22$ & 90.9 & $4 / 8$ & 50.0 & $24 / 30$ & 80.0 \\
\hline
\end{tabular}




\begin{tabular}{|c|c|c|c|c|c|c|c|}
\hline \multirow{5}{*}{$\begin{array}{l}\text { Knowledge on look back } \\
\text { period }\end{array}$} & Primary Syphilis & $18 / 22$ & 81.8 & $7 / 9$ & 77.7 & $25 / 31$ & 80.6 \\
\hline & Secondary syphilis & $18 / 21$ & 85.7 & $5 / 9$ & 55.5 & $23 / 30$ & 76.7 \\
\hline & Late syphilis & $6 / 22$ & 27.3 & $3 / 9$ & 33.3 & $9 / 21$ & 29.0 \\
\hline & Genital herpes & $18 / 21$ & 85.7 & $7 / 9$ & 77.7 & $25 / 30$ & 83.3 \\
\hline & Genital warts & $19 / 21$ & 90.5 & $8 / 9$ & 88.9 & $27 / 30$ & 90.0 \\
\hline \multirow{3}{*}{$\begin{array}{l}\text { What do you think about } \\
\text { partner notification in } \\
\text { managing patients with } \\
\text { bacterial STIs?" }\end{array}$} & Very important & $16 / 22$ & 72.7 & $4 / 9$ & 44.4 & $20 / 31$ & 64.5 \\
\hline & Important & $6 / 22$ & 27.3 & $3 / 9$ & 33.3 & $9 / 31$ & 29.0 \\
\hline & Not important & $0 / 22$ & 0.0 & $2 / 9$ & 22.2 & $2 / 31$ & 6.5 \\
\hline \multirow{3}{*}{$\begin{array}{l}\text { What do you think about } \\
\text { partner notification in } \\
\text { managing patients with } \\
\text { HIV infection? }\end{array}$} & Very important & $19 / 21$ & 90.5 & $9 / 10$ & 90.0 & $28 / 31$ & 90.3 \\
\hline & Important & $2 / 21$ & 9.5 & $1 / 10$ & 10.0 & $3 / 31$ & 9.7 \\
\hline & Not important & $0 / 21$ & 0.0 & $0 / 10$ & 0.0 & $0 / 31$ & 0.0 \\
\hline \multirow{7}{*}{$\begin{array}{l}\text { What do you consider as } \\
\text { the most effective } \\
\text { partner referral method? }\end{array}$} & Patient referral & $14 / 22$ & 63.6 & $6 / 9$ & 60.0 & $20 / 31$ & 64.5 \\
\hline & Provider referral & $3 / 22$ & 13.6 & $4 / 9$ & 44.4 & $7 / 31$ & 22.6 \\
\hline & Contract referral & $0 / 22$ & 0.0 & $1 / 9$ & 11.1 & $1 / 31$ & 3.2 \\
\hline & All three are the same & $3 / 22$ & 13.6 & $1 / 9$ & 11.1 & $4 / 31$ & 12.9 \\
\hline & $\begin{array}{l}\text { It varies with the diagnosis of index } \\
\text { patient }\end{array}$ & $5 / 22$ & 22.7 & $1 / 9$ & 11.1 & $6 / 31$ & 19.4 \\
\hline & It varies with other factors & $5 / 22$ & 22.7 & $0 / 9$ & 0.0 & $5 / 31$ & 16.1 \\
\hline & Don't know & $0 / 22$ & 0.0 & $0 / 9$ & 0.0 & $0 / 31$ & 0.0 \\
\hline \multirow{5}{*}{$\begin{array}{l}\text { What are the problems } \\
\text { that you encounter in } \\
\text { partner notifying services } \\
\text { in your clinic? }\end{array}$} & Inadequate public health staff & $8 / 16$ & 50.0 & $5 / 7$ & 71.4 & $13 / 23$ & 56.5 \\
\hline & $\begin{array}{l}\text { Inadequate training to public health } \\
\text { staff }\end{array}$ & $6 / 16$ & 37.5 & $0 / 7$ & 0.0 & $6 / 23$ & 26.1 \\
\hline & $\begin{array}{l}\text { Inadequate time for partner notifying } \\
\text { interviews }\end{array}$ & $10 / 16$ & 62.5 & $4 / 7$ & 57.1 & $14 / 23$ & 60.9 \\
\hline & $\begin{array}{l}\text { Frequent shortage of post cards/ } \\
\text { stamps/ envelopes }\end{array}$ & $0 / 16$ & 0.0 & $2 / 7$ & 28.6 & $2 / 23$ & 8.7 \\
\hline & Unavailability of a vehicle & $2 / 16$ & 12.5 & $3 / 7$ & 42.8 & $5 / 23$ & 21.7 \\
\hline \multirow{5}{*}{$\begin{array}{l}\text { When do you take the } \\
\text { consent of index patient } \\
\text { in partner tracing? }\end{array}$} & In all instances & $18 / 22$ & 81.8 & $8 / 8$ & 100.0 & $26 / 30$ & 86.7 \\
\hline & $\begin{array}{l}\text { When doing provider or contract } \\
\text { referral only }\end{array}$ & $1 / 22$ & 4.5 & $0 / 8$ & 0.0 & $1 / 30$ & 3.3 \\
\hline & $\begin{array}{l}\text { When the contact is marital or regular } \\
\text { partner only }\end{array}$ & $0 / 22$ & 0.0 & $0 / 8$ & 0.0 & $0 / 30$ & 0.0 \\
\hline & Only in HIV partner notification & $1 / 22$ & 4.5 & $0 / 8$ & 0.0 & $1 / 30$ & 3.3 \\
\hline & Don't know & $2 / 22$ & 9.1 & $0 / 8$ & 0.0 & $2 / 30$ & 6.7 \\
\hline \multirow{6}{*}{$\begin{array}{l}\text { In which condition/s do } \\
\text { you decide on visiting a } \\
\text { place to trace a partner? }\end{array}$} & $\begin{array}{l}\text { When only index patient volunteers to } \\
\text { accompany you to find the contact }\end{array}$ & $3 / 21$ & 14.3 & $2 / 10$ & 20.0 & $5 / 31$ & 16.1 \\
\hline & $\begin{array}{l}\text { When index patient not consents on } \\
\text { other modes of contact tracing }\end{array}$ & $3 / 21$ & 14.3 & $0 / 10$ & 0.0 & $3 / 31$ & 9.6 \\
\hline & $\begin{array}{l}\text { When other modes of contact tracing } \\
\text { were tried and failed }\end{array}$ & $10 / 21$ & 47.6 & $6 / 10$ & 60.0 & $16 / 31$ & 51.6 \\
\hline & $\begin{array}{l}\text { Whenever contact belongs to a high } \\
\text { risk group }\end{array}$ & $10 / 21$ & 47.6 & $5 / 10$ & 50.0 & $15 / 31$ & 48.4 \\
\hline & $\begin{array}{l}\text { When only index patient has early } \\
\text { syphilis or gonorrhoea }\end{array}$ & $1 / 21$ & 4.7 & $1 / 10$ & 10.0 & $2 / 31$ & 6.4 \\
\hline & Don't know & $2 / 21$ & 9.5 & $0 / 10$ & 0.0 & $2 / 31$ & 6.5 \\
\hline
\end{tabular}

$N=$ Total number answered, $n=$ Number correctly answered

Total of 1157 cases of partner notifiable diseases reported to selected clinics and distribution of partner notifiable diseases by clinic, sex, age groups and marital status are mentioned in table 4. 
Table 4: Distribution of partner notifiable STIs

\begin{tabular}{|c|c|c|c|c|c|c|}
\hline \multirow[t]{2}{*}{ Variable } & \multirow[t]{2}{*}{ Level } & Gonorrhoea & Syphilis & $\mathrm{NGI}$ & $\begin{array}{r}\text { Trichomoni } \\
\text {-sis }\end{array}$ & $\begin{array}{r}\text { Total } \\
\text { sample }\end{array}$ \\
\hline & & Number (\%) & Number (\%) & Number (\%) & Number (\%) & Number (\%) \\
\hline \multirow{6}{*}{$\begin{array}{l}\text { Partner notifiable STIs } \\
\text { by clinic }\end{array}$} & Ragama & $11(10.1)$ & $53(10.3)$ & 78 (15.1) & $0(0.0)$ & $142(12.3)$ \\
\hline & Kandy & $12(11.0)$ & $16(3.1)$ & $52(10.1)$ & $0(0.0)$ & $80(6.9)$ \\
\hline & Kalutara & $5(4.6)$ & $23(4.5)$ & 34 (6.6) & $0(0.0)$ & $62(5.3)$ \\
\hline & Gampaha & $4(3.7)$ & $11(2.1)$ & 90 (17.5) & $0(0.0)$ & $105(9.1)$ \\
\hline & Colombo & 77 (70.6) & $412(80.0)$ & $261(50.7)$ & $18(100.0)$ & 768 (66.4) \\
\hline & Total & $109(100.0)$ & $515(100.0)$ & $515(100.0)$ & $18(100.0)$ & $1157(100)$ \\
\hline \multirow{3}{*}{$\begin{array}{l}\text { Partner notifiable STIs } \\
\text { by sex of the patient }\end{array}$} & Male & $98(90.0)$ & $347(67.4)$ & $177(34.4)$ & $0(0.0)$ & $622(53.8)$ \\
\hline & Female & $11(10.0)$ & $168(32.6)$ & $338(65.6)$ & $18(100.0)$ & $535(46.2)$ \\
\hline & Total & $109(100.0)$ & $515(100.0)$ & $515(100.0)$ & $18(100.0)$ & $1157(100)$ \\
\hline \multirow{4}{*}{$\begin{array}{l}\text { partner notifiable STIS } \\
\text { by age group }\end{array}$} & $15-24$ & $27(24.8)$ & $80(15.5)$ & $107(20.8)$ & 3 (16.7) & $217(18.8)$ \\
\hline & $25-49$ & $78(71.5)$ & $359(69.7)$ & $372(72.2)$ & $13(72.2)$ & $822(71.0)$ \\
\hline & $\geq 50$ & $4(3.7)$ & $76(14.8)$ & $36(7.0)$ & $2(11.1)$ & $118(10.2)$ \\
\hline & Total & $109(100.0)$ & $515(100.0)$ & $515(100.0)$ & $18(100.0)$ & $1157(100)$ \\
\hline \multirow{4}{*}{$\begin{array}{l}\text { Partner notifiable STIs } \\
\text { by marital status }\end{array}$} & Single & $60(55.0)$ & $185(35.9)$ & $118(22.9)$ & $4(0.3)$ & $367(31.7)$ \\
\hline & M/LT & $45(41.3)$ & $268(52.0)$ & $322(27.8)$ & $8(0.7)$ & $643(55.6)$ \\
\hline & W/S/D & $4(3.7)$ & $62(12.1)$ & $75(6.5)$ & $6(0.5)$ & 147(12.7) \\
\hline & Total & $109(100.0)$ & $515(100.0)$ & $515(44.5)$ & $18(1.6)$ & $1157(100)$ \\
\hline
\end{tabular}

Out of the 19 variables considered in the case control study (component 3), sex, age category, marital status, level of education, state of employment, reason for attendance, presence of symptoms, previous history of STIs, diagnosis of gonorrhoea or syphilis, condom use in last 3 months, condom use at last sexual exposure and sexuality were not significantly associated with the compliance with partner notification. Table 5 indicates the variables that had a significant association.

Table 5: Summary of variables found to be significant in univariate analysis between cases and controls

\begin{tabular}{|l|r|r|r|}
\hline Variable & $\begin{array}{r}\text { Factors associated with } \\
\text { compliance }\end{array}$ & $\begin{array}{r}\text { Statistical test } \\
\text { Odds Ratio (OR) }\end{array}$ & $\begin{array}{r}95 \% \text { Confidence } \\
\text { interval }\end{array}$ \\
\hline Marital status & Being married/cohabitant & 5.9 & $3.0-11.7$ \\
\hline $\begin{array}{l}\text { Type of sexual partner during last 12 } \\
\text { months }\end{array}$ & $\begin{array}{r}\text { Regular partner/s during last } \\
12 \text { months }\end{array}$ & 3.4 & $1.8-6.4$ \\
\hline $\begin{array}{l}\text { Time of last sexual } \\
\text { exposure }\end{array}$ & Within 4 weeks & 2.7 & $1.4-5.1$ \\
\hline Last sexual partner & Marital/ regular partner & 5.5 & $2.9-10.4$ \\
\hline Age at coitarche & $\geq 19$ years & 2.4 & $1.3-4.6$ \\
\hline
\end{tabular}

Since the significance could have been due to confounding effects, binary logistic regression analysis was done. Significant variables given in table 5 and four other likely important variables were included in binary logistic model. Table 6 shows the last step of the binary logistic regression analysis done using backward logistic regression option. Following four variables were statistically significantly associated with the compliance with partner notification. a) Being married/co-habitant (OR=2.54, 95\% $\mathrm{Cl} 0.93-$ 6.3)

b) $\mathrm{NGI}(\mathrm{OR}=0.4,95 \% \mathrm{Cl}$ 0.19-0.89)-Showed a negative association

c) Last sexual partner being marital / regular partner (OR=3.57, 95\% Cl 1.4-8.9)

d) Age at coitarche $\geq 19$ years (OR=2.39, 95\% Cl 1.24.9) 
Table 6: Binary logistic regression analysis

\begin{tabular}{|c|c|c|c|c|c|c|c|c|}
\hline & \multirow[b]{2}{*}{ B } & \multirow[b]{2}{*}{ S.E. } & \multirow[b]{2}{*}{ Wald } & \multirow[b]{2}{*}{$\mathrm{df}$} & \multirow[b]{2}{*}{ Sig. } & \multirow{2}{*}{$\begin{array}{r}\operatorname{Exp}(\mathrm{B}) \\
\text { (Odd ratio) } \\
\end{array}$} & \multicolumn{2}{|c|}{$\begin{array}{r}95 \% \\
C I \text { for } \operatorname{EXP}(B) \\
\end{array}$} \\
\hline & & & & & & & Lower & Upper \\
\hline Married/co-habitant & .933 & .489 & 3.642 & 1 & .056 & 2.54 & 0.97 & 6.63 \\
\hline Diagnosis NGI & -.893 & .398 & 5.038 & 1 & .025 & .40 & 0.19 & .89 \\
\hline $\begin{array}{l}\text { Last sexual partner being } \\
\text { maital/regular partner }\end{array}$ & 1.275 & .470 & 7.348 & 1 & .007 & 3.57 & 1.42 & 8.99 \\
\hline Age at coitarche & .874 & .370 & 5.571 & 1 & .018 & 2.39 & 1.16 & 4.95 \\
\hline
\end{tabular}

Standard partner notification indices that can be used to evaluate the performance of partner notification process were calculated in component 4 (table 7). Total number of index patients with partner notifiable diseases reported in 2012 was 745 . Total number of contacts who attended the clinic after the first interview was 62. Majority of them (85.4\%) have attended within 28 days while $56.4 \%$ out of the attendees have done so within the first week of interview.

Table 7: Partner notification indices

\begin{tabular}{|c|c|c|c|c|}
\hline Index & Description of numerator and denominator & Values & & Index value \\
\hline \multirow{2}{*}{$\begin{array}{l}\text { 1. Percentage of index cases that } \\
\text { were offered at least one } \\
\text { interview for partner notification }\end{array}$} & $\begin{array}{l}\text { Number of index cases with partner notifiable } \\
\text { infections, interviewed at least once }\end{array}$ & 229 & \multirow{2}{*}{$\times 100$} & \multirow{2}{*}{$30.7 \%$} \\
\hline & Number of index patients with partner notifiable STIs & 745 & & \\
\hline \multirow{2}{*}{$\begin{array}{l}2.1 \text { percentage of index cases } \\
\text { having documented attendance } \\
\text { for all contacts }\end{array}$} & $\begin{array}{l}\text { Number of index cases who has documented } \\
\text { attendance for all contacts }\end{array}$ & 60 & \multirow{2}{*}{$\times 100$} & \multirow{2}{*}{$8.05 \%$} \\
\hline & Number of index patients with partner notifiable STIs & 745 & & \\
\hline \multirow{2}{*}{$\begin{array}{l}\text { 2.2.percentage of index cases } \\
\text { having documented status of } \\
\text { infection for all contacts }\end{array}$} & $\begin{array}{l}\text { Number of index cases who has documented status } \\
\text { of infection for all contacts }\end{array}$ & 60 & \multirow{2}{*}{$\times 100$} & \multirow[b]{2}{*}{$8.05 \%$} \\
\hline & Number of index patients with partner notifiable STIs & 745 & & \\
\hline \multirow{2}{*}{$\begin{array}{l}2.3 \text { Percentage of index cases } \\
\text { having documented status of } \\
\text { treatment for all contacts }\end{array}$} & $\begin{array}{l}\text { Number of index cases who has documented status } \\
\text { of treatment for all contacts }\end{array}$ & 60 & \multirow{2}{*}{$\times 100$} & \multirow[b]{2}{*}{$8.05 \%$} \\
\hline & Number of index patients with partner notifiable STIs & 745 & & \\
\hline \multirow[t]{2}{*}{$\begin{array}{l}\text { 3.Verified NGI partner } \\
\text { notification index }\end{array}$} & $\begin{array}{l}\text { Number of all contacts of index patients with NGIs, } \\
\text { whose attendance was documented as verified, } \\
\text { within four weeks of the first interview }\end{array}$ & 14 & \multirow[t]{2}{*}{$\mathrm{X} 100$} & \multirow[t]{2}{*}{$5.3 \%$} \\
\hline & Number of index patients with NGls & 261 & & \\
\hline \multirow{2}{*}{$\begin{array}{l}\text { 4.Contacts infected index for } \\
\text { gonorrhoea }\end{array}$} & $\begin{array}{l}\text { Number of contacts infected with gonorrhoea } \\
\text { detected by partner notification process }\end{array}$ & 3 & \multirow[b]{2}{*}{$\mathrm{X} 100$} & \multirow[b]{2}{*}{$8.1 \%$} \\
\hline & $\begin{array}{l}\text { Number of contacts of index patients with } \\
\text { gonorrhoea on whom partner notification was } \\
\text { initiated }\end{array}$ & 37 & & \\
\hline \multirow{2}{*}{$\begin{array}{l}\text { Contacts infected index for } \\
\text { syphilis }\end{array}$} & $\begin{array}{l}\text { Number of contacts infected with syphilis detected } \\
\text { by partner notification process }\end{array}$ & 9 & \multirow[b]{2}{*}{$\mathrm{X} 100$} & \multirow[b]{2}{*}{$6.5 \%$} \\
\hline & $\begin{array}{l}\text { Number of contacts of index patients with syphilis on } \\
\text { whom partner notification was initiated }\end{array}$ & 139 & & \\
\hline \multirow[b]{2}{*}{ Contacts infected index for NGIs } & $\begin{array}{l}\text { Number of contacts infected with NGI detected by } \\
\text { partner notification process }\end{array}$ & 5 & \multirow[b]{2}{*}{$\mathrm{X} 100$} & \multirow[b]{2}{*}{$9.1 \%$} \\
\hline & $\begin{array}{l}\text { Number of contacts of index patients with NGlon } \\
\text { whom partner notification was initiated }\end{array}$ & 55 & & \\
\hline \multirow{2}{*}{ Contact index } & Number of contacts obtained & \multirow{2}{*}{\multicolumn{3}{|c|}{$\begin{array}{l}\text { This could not be calculated as } \\
\text { no data were recorded as } \\
\text { number of contacts obtained } \\
\text { in an interview. }\end{array}$}} \\
\hline & $\begin{array}{l}\text { Number of interviews held for index patients with } \\
\text { partner notifiable STIs }\end{array}$ & & & \\
\hline
\end{tabular}




\section{Discussion}

Despite the mandatory training given to all concerned staff at the beginning of their carrier in a STD clinic and reasonably long service period, basic knowledge of partner notification among them was not up to an acceptable level.

All interviewed staff knew that gonorrhoea, early syphilis and chlamydia infection are partner notifiable but some misidentified bacterial vaginosis (20\%) and vaginal candidiasis $(14 \%)$ as partner notifiable. Only61.3\%were aware that late syphilis is partner notifiable. Knowledge on look back period was unsatisfactory and $17 \%$ and $10 \%$ respectively were unaware that look back period is irrelevant to genital herpes and genital warts. Only nearly $1 / 3$ knew the correct look back period for late syphilis.

Some participants (6.5\%) have stated that partner notification is not important in bacterial infections. More than half of the sample has identified limited time and manpower as the main problems they encounter in partner notification in their clinics.

Prevalence of syphilis among males was nearly twice as that of females while the prevalence of $\mathrm{NGI}$ among males was nearly half as that among females. In contrast, the WHO estimate for the region (5) states that the prevalence of chlamydia infections is nearly twice among males (chlamydia is considered to cause $70 \%$ of non gonococcal urethritis) as that of females but the estimation for the prevalence for syphilis is the same for both sexes. The higher prevalence of $\mathrm{NGI}$ among females in the present study can be explained by the mostly subjective diagnosis among females in contrast to the objective diagnosis among males in Sri Lanka.

Although it is reasonable to anticipate an inverse relationship between the prevalence of STIs and the educational level, it was striking to observe that both groups of patients who had no schooling and who had diploma or degree had almost the same proportion (4\%) of STIs.

Being married or co-habitant was significantly associated with compliance with partner notification. The reasons for this were not assessed in the present study. A systematic literature review in Africa, Asia and Latin American and Caribbean countries has revealed that major barriers for notifying casual and commercial partners were nontraceability of them and that men had no further plans to continue sexual relationships with them. (6)

In this study, gonorrhoea and syphilis did not have an association with compliance. But the patients who had NGI were 2.5 times less likely to be compliant with partner notification than those who did not have NGI.

Last sexual exposure within four weeks was significantly associated with compliance, after univariate analysis but after binary logistic regression that association was not found to be significant. An observational study among patients with gonorrhoea or Chlamydia infection in Netherlands indicated that last exposure less than 1 week has been significantly associated with the success of partner notification Vs last exposure more than 1 month back. (7)

Percentage of index cases that had at least one interview for partner notification was $30.7 \%$.In an audit done by the principal investigator in a sexual health clinic in Portsmouth, UK (8) $73 \%$ of the index patients have been interviewed by the second week after the diagnosis. According to the British statement on partner notification in 2012 (1) the standard performance of this index is $97 \%$. In the present study, some index patients have been given contact slips for partner notification by the MOs or the public health staff without filling $\mathrm{H} 1205$. Hence the actual number of the index patients that were interviewed may be higher than that of calculated. 
The percentage of index cases with the outcome of an agreed contact action (attendance, result of testing and treatment for the contact) documented for all contacts was $8.1 \%$. In the above audit (8) the percentages of index cases with documented attendance, status of infection and status of treatment were 46.1, 10.6 and 48.1 respectively. The standard percentage for index patients who have documented outcomes for agreed contact action for all contacts is $97 \%$ according to the British statement. (1) Only two contact actions, patient and provider referral are mentioned in the recording formats used in Sri Lanka. Contract referral and no action, are not mentioned in those formats further lowering the outcomes.

Verified NGI partner notification index was 0.05 contacts per case. The standard for verified chlamydial partner notification is at least 0.4 contacts per index case for all clinics in UK. (1) This comparison is justifiable since more than $70 \%$ of the NGIs in males are due to Chlamydia infection. (2)

British statement mentions that outcomes of all agreed contact actions should be completed by 28 days. Anyhow in this study, six months were allowed after the first interview, for partners to be brought before closing each scenario but allowing a longer period did not make a better outcome.

Although the values obtained in this study cannot be compared with the British standards or the values from any developed country, these results are below satisfactory level.

\section{Conclusions}

The level of Knowledge, attitude and practices on partner notification among clinic staff was unsatisfactory. The percentage of index cases interviewed was low (30\%) probably leaving a significant number of contacts unattended. Patient compliance on partner notification was significantly associated with being married/co-habitant, last sexual exposure with regular partner, coitarche $\geq 19$ years and NGI. Documentation relevant to partner notification was unsatisfactory.

It is advisable to well train the staff on partner notification and to introduce one format to record all data in partner notification instead of using many formats. Additional information such as number of partners elicited, agreed contact action, status of notification, detailed outcome of an agreed contact action should be included in the new format.

Provider or contract referral was never been observed within the study. More attention should be paid to improve those contact actions by providing the STD clinics with essential resources such as transport facility and funding.

\section{References}

1. BASHH statement for partner notification in STIS, 2012

2. Centres for Disease Control and Prevention: "Sexual and reproductive health of persons aged 10-24 years-United States, 2002-2007," MMWR Surveillance Summaries, vol. 58, pp. 1-58, 2009.

3. User Manual for STI Patient Information Management System; 2008 June; NSACP

4. James J. Schlesselman Professor; Department of Clinical Epidemiology and Preventive Medicine University of Pittsburgh School of Medicine; CaseControl Studies: Design, Conduct, Analysis: Monographs in epidemiology and biostatistics

5. Global incidence and prevalence of selected curable STIs:WHO; 2008 Geneva

6. Sexually Transmitted Infections Case Initiated Partner Notification: Best Approaches in Winnipeg, Manitoba; April, 2011

7. European Centre for Disease Prevention and Control; Technical report: Public health benefits of partner notification for sexually transmitted infections and HIV; 2013

8. Wijayawickrama D.N. An audit on partner notification among patients attending the sexual health clinic, St Maries Hospital, Portsmouth, UK; 2014 